\title{
The impact of sarcopenia on the outcome of patients with cirrhosis with and without hepatocellular carcinoma who undergo liver transplantation
}

\author{
Francesca D’Arcangelo1,*, Alberto Zanetto ${ }^{1, *}$, Camillo Aliberti ${ }^{2}$, Sarah Shalaby ${ }^{1}$, Monica Pellone ${ }^{1}$, Salvatore \\ Stefano Sciarrone ${ }^{1}$, Chiara Becchetti ${ }^{1}$, Alberto Ferrarese ${ }^{1}$, Martina Gambato ${ }^{1}$, Francesco Paolo Russo ${ }^{1}$, \\ Giacomo Germani ${ }^{1}$, Marco Senzolo ${ }^{1}$, Alessandro Vitale ${ }^{3}$, Umberto Cillo ${ }^{3}$, Patrizia Burra ${ }^{1}$

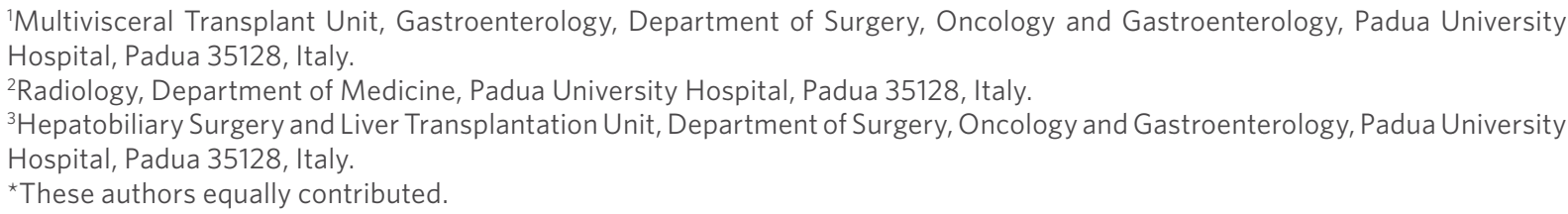

Correspondence to: Prof. Patrizia Burra, Multivisceral Transplant Unit, Gastroenterology, Department of Surgery, Oncology and Gastroenterology, Padua University Hospital, Via Giustiniani 2, Padua 35128, Italy. E-mail: burra@unipd.it

How to cite this article: D'Arcangelo F, Zanetto A, Aliberti C, Shalaby S, Pellone M, Sciarrone SS, Becchetti C, Ferrarese A, Gambato M, Russo FP, Germani G, Senzolo M, Vitale A, Cillo U, Burra P. The impact of sarcopenia on the outcome of patients with cirrhosis with and without hepatocellular carcinoma who undergo liver transplantation. Hepatoma Res 2021;7:4.

http://dx.doi.org/10.20517/2394-5079.2020.109

Received: 18 Sep 2020 First Decision: 10 Oct 2020 Revised: 26 Oct 2020 Accepted: 5 Nov 2020 Published: 7 Jan 2021

Academic Editor: Guido Guenther Gerken Copy Editor: Cai-Hong Wang Production Editor: Jing Yu

\begin{abstract}
Background: The impact of sarcopenia on the outcome of patients with cirrhosis who undergo liver transplantation (LT) has been analysed in heterogeneous cohorts with mixed results. We sought to determine the prevalence and the impact of pre-LT sarcopenia on morbidity and mortality after LT in a cohort of patients with cirrhosis with and without hepatocellular carcinoma (HCC).
\end{abstract}

Methods: Patients with cirrhosis who underwent LT between 2010 and 2016 at Padua University Hospital were retrospectively evaluated. Using image software analysis, cross-sectional area of skeletal muscle at 3rd lumbar vertebra was measured and skeletal muscle index (SMI) was calculated. Sarcopenia was defined by $\mathrm{SMI}<50 \mathrm{~cm}^{2} / \mathrm{m}^{2}$ in males and $<39 \mathrm{~cm}^{2} / \mathrm{m}^{2}$ in females, respectively. Primary outcome was post-LT survival. Secondary outcomes included hospitalization length and post-LT complications. 
Results: 197 patients were included, of whom, 122 (62\%) had sarcopenia. Demographics and severity of cirrhosis were comparable in patients with $v s$. without sarcopenia. Overall survival was similar between the groups. When survival analysis was adjusted for severity of liver disease, sarcopenia was associated with a significantly reduced survival in decompensated ( $80 \%$ vs. $91 \%, 1$-year post-LT; $P=0.04$ ) but not in compensated ( $93 \%$ vs. $90 \%$, 1-year post-LT; $P=0.7$ ) patients. In patients with HCC, sarcopenia was associated with a trend towards lower survival but only in those with HCC beyond Milan criteria. Among secondary outcomes, bacterial infections were more frequent in patients with vs. without sarcopenia (50\% vs. 35\%; $P=0.02)$, whereas hospitalization length and other complications were comparable between the groups.

Conclusion: Sarcopenia is a common finding in patients awaiting LT and, in those with decompensated cirrhosis, it is associated with reduced survival after transplantation.

Keywords: Sarcopenia, cirrhosis, hepatocellular carcinoma, liver transplantation, survival

\section{INTRODUCTION}

Sarcopenia is defined as the generalized loss of skeletal muscles mass, strength, and function ${ }^{[1]}$. It is reported in approximately $50 \%$ of patients with cirrhosis awaiting liver transplantation (LT), with a relatively higher prevalence in male compared to female candidates ${ }^{[2-9]}$.

Recent evidence suggests that, in patients with cirrhosis, sarcopenia is independently correlated with increased risks of liver decompensation and mortality, both before and after transplantation ${ }^{[10-12]}$.

On the other hand, most studies looking at the effects of sarcopenia on post-transplant outcomes have included heterogeneous cohorts of patients with no adjustments for severity of cirrhosis (compensated $v s$. decompensated) and/or indication for transplantation ${ }^{[8,13-20]}$. This led to rather controversial results with some studies that found sarcopenia to be associated with increased risk of post-transplant mortality and others that did not ${ }^{[8,13-20]}$. Therefore, whether and how sarcopenia impacts post-LT survival remains unclear.

Understanding the effect of sarcopenia on morbidity and mortality after liver transplantation would have important implications for the management of liver transplant candidates and recipients ${ }^{[2,22]}$. The goals of our retrospective study were (1) to evaluate prevalence and characteristics of sarcopenia in a large cohort of patients with cirrhosis with and without hepatocellular carcinoma (HCC) awaiting LT at a first level center; and (2) to determine the impact of pre-transplant sarcopenia on morbidity and mortality after LT in these patients.

\section{METHODS}

\section{Patient selection}

Adult (> 18 years) patients with cirrhosis who underwent LT between 2010 and 2016 at Padua University Hospital were retrospectively evaluated to determine eligibility to be included. The diagnosis of cirrhosis was confirmed with available data including histology, radiology, laboratory, and clinical assessment. Decompensation was defined by the presence or history of clinically evident decompensating events (i.e., ascites, variceal haemorrhage, and hepatic encephalopathy ${ }^{[23,24]}$. Diagnosis of HCC was based on guidelines from the European Association for the Study of Liver ${ }^{[25]}$. Patients with HCC were further divided into patients with HCC within Milan criteria (MC) and patients with HCC beyond $\mathrm{MC}^{[26]}$. Milan criteria were defined as follows: single nodule with diameter $<5 \mathrm{~cm}$ or no more than 3 nodules with each nodule $<3 \mathrm{~cm}$, without angioinvasion and with no extrahepatic metastasis ${ }^{[26]}$. 


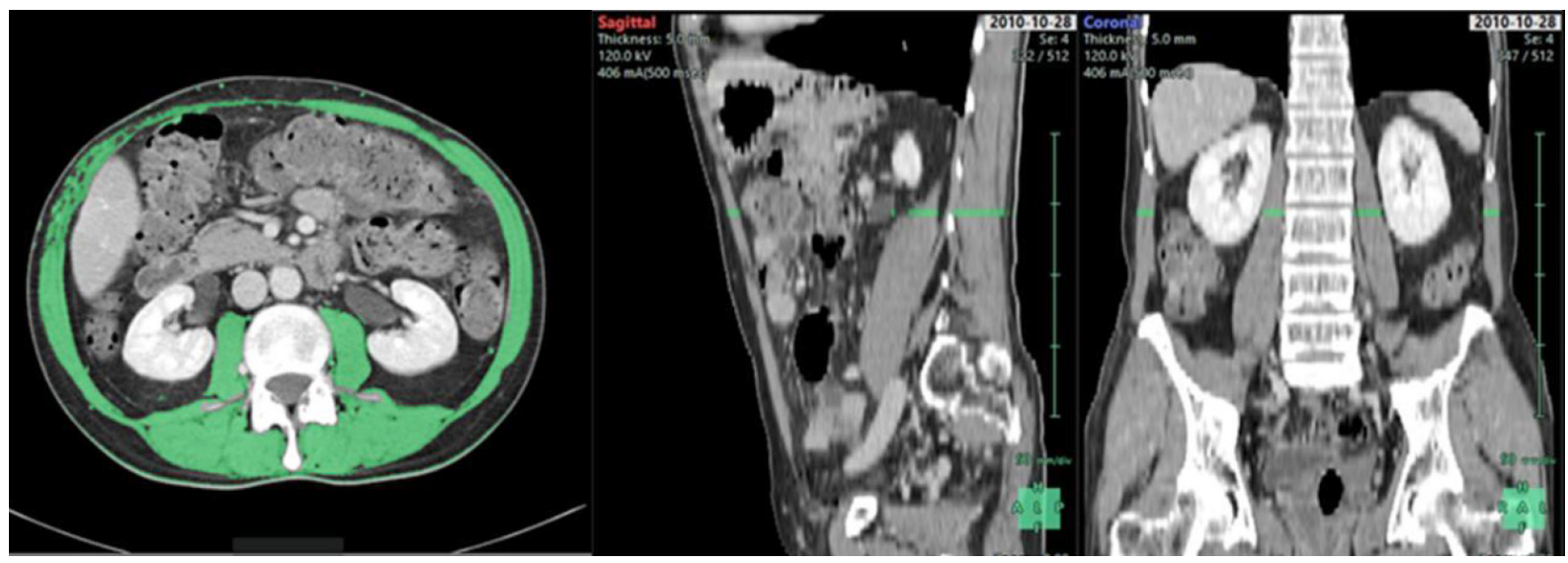

Figure 1. Assessment of sarcopenia in patients with cirrhosis. Using imagine software analysis (Fujifilm Synapse 3D ${ }^{\mathrm{TM}}$ ), the total crosssectional muscle area (CSMA, $\left.\mathrm{cm}^{2}\right)$ at level of 3rd lumbar vertebra was calculated. CSMA was then divided per patient's height to calculate the skeletal muscle index $(\mathrm{SMI})\left(\mathrm{cm}^{2} / \mathrm{m}^{2}\right)$. Sarcopenia was defined by $\mathrm{SMI}<50 \mathrm{~cm}^{2} / \mathrm{m}^{2}$ in male patients and $<39 \mathrm{~cm}^{2} / \mathrm{m}^{2}$ in female patients, respectively

At screening, patient's medical records, past medical history, and laboratory data were reviewed for the following exclusion criteria: absence of abdominal computed tomography scan (CT) within the 6 months prior to LT, more than one LT, and combined liver-kidney transplantation. Upon having determined eligibility to be included, patients were categorized into cases (with sarcopenia) and controls (without sarcopenia).

\section{Assessment of abdominal muscle mass and definition of sarcopenia}

Pre-transplant abdominal skeletal muscle mass was assessed by evaluating the last available CT scan within 6 months prior to LT [Figure 1]. Using image software analysis (Fujifilm Synapse $3 \mathrm{D}^{\mathrm{TM}}$ ), one expert operator calculated the total cross-sectional muscle area $\left(\mathrm{CSMA}, \mathrm{cm}^{2}\right)$ at level of the 3 rd lumbar vertebra (L3) [Figure 1]. CSMA was then divided per patient's height squared to calculate the skeletal muscle index (SMI) $\left(\mathrm{cm}^{2} / \mathrm{m}^{2}\right)$.

Per previously established cut-offs in patients with cirrhosis awaiting $\mathrm{LT}^{[3]}$, sarcopenia was defined by SMI $<50 \mathrm{~cm}^{2} / \mathrm{m}^{2}$ in males and $<39 \mathrm{~cm}^{2} / \mathrm{m}^{2}$ in females.

\section{Study design and data collection}

This was a single-center, retrospective, case-control study approved by the Padua University Hospital Ethical Committee (\#AOP/0564). The study was conducted in compliance with the Declaration of Helsinki and a waiver for informed consent was obtained for this retrospective chart review.

Pre-transplant variables collected from the medical records included demographics and body mass index, diabetes mellitus, aetiology of cirrhosis, duration of wait list time, presence of portal vein thrombosis, Child class, and MELD (Model for End-Stage Liver Disease) score at time of LT. In patients with HCC, the number and size of nodules at the last CT scan prior to LT were also collected.

The following post-transplant outcomes were evaluated: length of hospitalization (both in the intensive care unit and in total), rates of primary non-function (PNF), acute and chronic rejection, early (within 30-day) infections (i.e., viral, bacterial, and fungal), any biliary complications, and de novo malignancy. Patient's survival was recorded at the last available follow-up.

\section{Study objectives}

Primary objectives of this study were (1) to determine the prevalence of sarcopenia in a large cohort of 
patients with and without HCC awaiting LT at a first-level center; and (2) to assess the impact of sarcopenia on patient survival after LT in these patients.

Secondary objective of this study was to assess the impact of sarcopenia on post-LT length of hospitalization and risk of short- and medium-term complications.

Because severity of cirrhosis may influence prevalence and impact of sarcopenia ${ }^{[6]}$, we performed a post-hoc analysis in compensated (Child A) and decompensated (Child B/C) patients separately (with vs. without sarcopenia).

Because the muscle mass may vary over a 6 months period in patients with cirrhosis, we performed a post-hoc analysis in patients who had CT scan within 3 months prior to LT and in patients who had CT scan between 3 months and 6 months prior to LT, separately.

In the subgroup of patients with HCC, we hypothesized that pre-LT tumour burden might influence the impact of sarcopenia on post-LT outcome. Hence, we analysed survival in patients with HCC beyond Milan criteria and in patients with HCC within Milan criteria separately (with $v s$. without sarcopenia) ${ }^{[26]}$.

\section{Statistical analysis}

Values for continuous variables are presented as mean \pm standard deviation. Categorical-nominal variables are presented as frequencies. For subgroup comparisons, quantitative variables were compared using Student's $t$-test or Mann-Whitney $U$ test, and categorical variables using $\chi^{2}$ or Fisher's exact tests, as appropriate. Survival curves were estimated with Kaplan-Meier method and compared with log rank test. All tests were 2 -tailed, and $P$-value $<0.05$ was considered statistically significant. Statistical analysis was performed using SPSS (version 25.0).

\section{RESULTS}

\section{Demographics and prevalence of sarcopenia}

Of 475 patients who were screened for eligibility, 197 were included (male/female 153/44; mean age 57 years). Reason for exclusion were as follows: no CT scan in the 6 months prior to LT $(n=235)$, more than one LT $(n=40)$, and combined liver-kidney transplantation $(n=3)$.

Overall, the most common aetiology of cirrhosis was hepatitis C virus (HCV) infection (42\%), followed by alcoholic (19\%), and combined HCV + alcoholic (12\%) liver disease. In approximately $70 \%$ of these patients, HCC was the indication for LT. In patients with HCC, MELD score was comparable between those with HCC within compared to those with HCC beyond MC (12 vs. 11, respectively). On the other hand, patients with HCC beyond MC tended to be more compensated compared with patients with HCC within MC (Child A: 58\% vs. 39\% and Child B/C $42 \%$ vs. $61 \%$ in patients with HCC beyond vs. within MC, respectively; $P=0.05)$.

Prevalence of sarcopenia was $62 \%$, being relatively higher in male compared to female patients (65\%vs. $50 \%$, respectively). Overall, prevalence of sarcopenia increased in parallel with severity of liver dysfunction (55\% in Child A, 63\% in Child B, and $72 \%$ in Child C patients). However, in sex-stratified analysis, this association was significant in male (55\% in Child A, $65 \%$ in Child B, and $80 \%$ in Child C patients; $P=0.03$ ) but not in female (56\% in Child A, $56 \%$ in Child B, and $39 \%$ in Child C patients; $P=0.5$ ) candidates. No association was found between prevalence of sarcopenia and aetiology of cirrhosis ( $60 \%$ vs. $63 \%$ vs. $65 \%$ in alcoholic, HCV, and metabolic patients, respectively), nor between sarcopenia and presence of HCC (55\% vs. $65 \%$ in patients with and without HCC, respectively). In the subgroup of patients with HCC, there was a trend towards a higher prevalence of sarcopenia in those with HCC beyond Milan criteria compared to 
Table 1. Baseline characteristics of the study cohort

\begin{tabular}{|c|c|c|c|}
\hline & $\begin{array}{l}\text { Patients with sarcopenia } \\
(n=122)\end{array}$ & $\begin{array}{l}\text { Patients without sarcopenia } \\
(n=75)\end{array}$ & $P$ values \\
\hline Gender, $n(\%)$ & & & 0.06 \\
\hline Male & $100(82 \%)$ & $53(70 \%)$ & \\
\hline Female & $22(18 \%)$ & $22(30 \%)$ & \\
\hline Age at LT (years) & $57[8]$ & $57[7]$ & 0.8 \\
\hline Etiology of cirrhosis & & & 0.9 \\
\hline $\mathrm{HCV}$ & $51(41 \%)$ & $33(44 \%)$ & \\
\hline Alcohol & $24(20 \%)$ & $14(18 \%)$ & \\
\hline HBV & $12(10 \%)$ & $8(11 \%)$ & \\
\hline $\mathrm{HCV}+$ alcohol & $15(12 \%)$ & $8(11 \%)$ & \\
\hline Metabolic & $12(10 \%)$ & $7(9 \%)$ & \\
\hline Other & $8(7 \%)$ & $5(7 \%)$ & \\
\hline $\mathrm{HCC}, n(\%)$ & $83(68 \%)$ & $59(78 \%)$ & 0.1 \\
\hline Body mass index & $24.4[3.9]$ & $27.3[3.8]$ & 0.001 \\
\hline Diabetes mellitus (\%) & $32(26 \%)$ & $19(25 \%)$ & 0.8 \\
\hline Child Class* & & & 0.3 \\
\hline $\mathrm{A}, n(\%)$ & $36(29 \%)$ & $29(39 \%)$ & \\
\hline $\mathrm{B}, n(\%)$ & $42(34 \%)$ & $25(33 \%)$ & \\
\hline $\mathrm{C}, n(\%)$ & $43(36 \%)$ & $20(27 \%)$ & \\
\hline MELD at time of LT & $18[9]$ & $15[9]$ & 0.04 \\
\hline $\mathrm{PVT}, n(\%)$ & $23(19 \%)$ & $17(21 \%)$ & - \\
\hline $\begin{array}{l}\text { Interval between radiological assessment } \\
\text { of sarcopenia and LT (months) }\end{array}$ & $3[2.5]$ & $2.9[3.4]$ & 0.3 \\
\hline Wait list time (months) & $9.4[15]$ & $9[11]$ & 0.8 \\
\hline
\end{tabular}

Continuous variable expressed as mean [SD], categorical data expressed as frequency and percentage. LT: liver transplantation; HCV: hepatitis C virus; HBV: hepatitis B virus; HCC: hepatocellular carcinoma; MELD: model for end-stage liver disease; PVT: portal vein thrombosis. ${ }^{*}$ In Child $\mathrm{A}$ and $\mathrm{B} / \mathrm{C}$ class, $\mathrm{HCV}$ was the indication for transplantation in $53 \%$ and $41 \%$ of patients, respectively

those with HCC within Milan criteria; however the difference was not statistically significant (66\% vs. 49\%; $P=0.1)$.

As shown in Table 1, demographics, indications for LT, history of decompensation, and Child class were comparable between patients with and without sarcopenia [Table 1]. On the other hand, MELD score at LT was significantly higher in patients with compared to those without sarcopenia $(18 \mathrm{vs} .15$, respectively; $P=$ $0.04)$.

\section{Patient survival and post-transplant complications in patients with and without sarcopenia}

Mean duration of follow up was 48 months and 46 months in patients with and without sarcopenia, respectively $(P=0.7)$.

The most commonly used immunosuppressive regimen was tacrolimus \pm steroids ( $45 \%$ in patients with $v s$. $54 \%$ in patients without sarcopenia), followed by tacrolimus + everolimus \pm steroids ( $22 \%$ in patients with vs. $29 \%$ in patients without sarcopenia), and tacrolimus + mycophenolate \pm steroids ( $11 \%$ in patients with vs. $12 \%$ in patients without sarcopenia), respectively $(P=0.8 ; P=0.6$; and $P=0.5$, respectively).

Overall, patient's survival at 1, 3, and 5 years after LT was comparable between the study groups [Figure 2]. However, when the analysis was adjusted for the severity of underlying liver disease, those with decompensated cirrhosis (Child $\mathrm{B} / \mathrm{C})$ and sarcopenia $(n=85)$ showed a significantly reduced survival compared with those without sarcopenia $(n=47)$, whereas in compensated patients (Child A) no difference was found between sarcopenic $(n=36)$ and non-sarcopenic $(n=29)$ recipients [Figure 3]. 


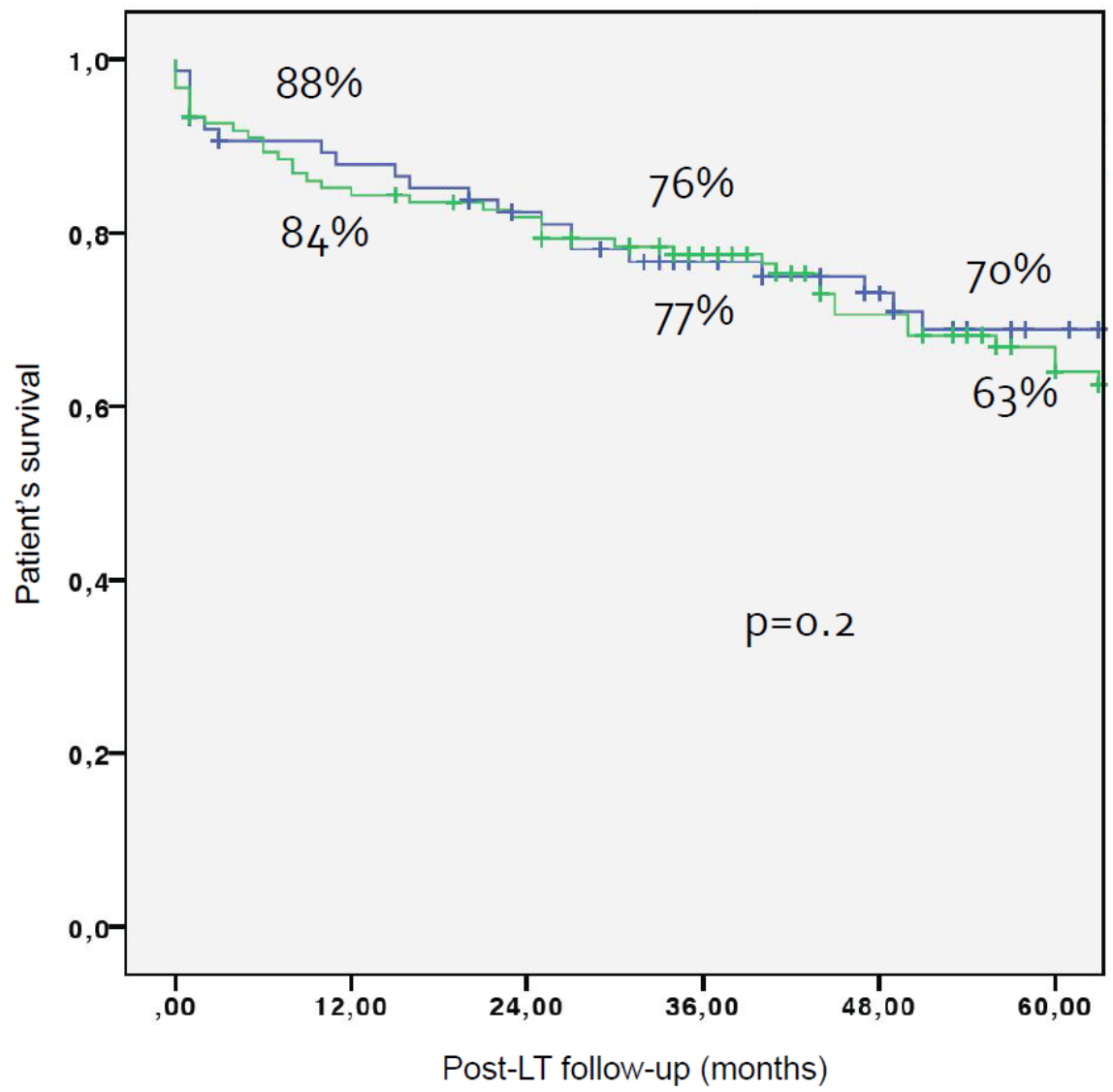

Figure 2. Post-transplant survival after transplantation was comparable in patients with (green line) vs. without (blue line) sarcopenia
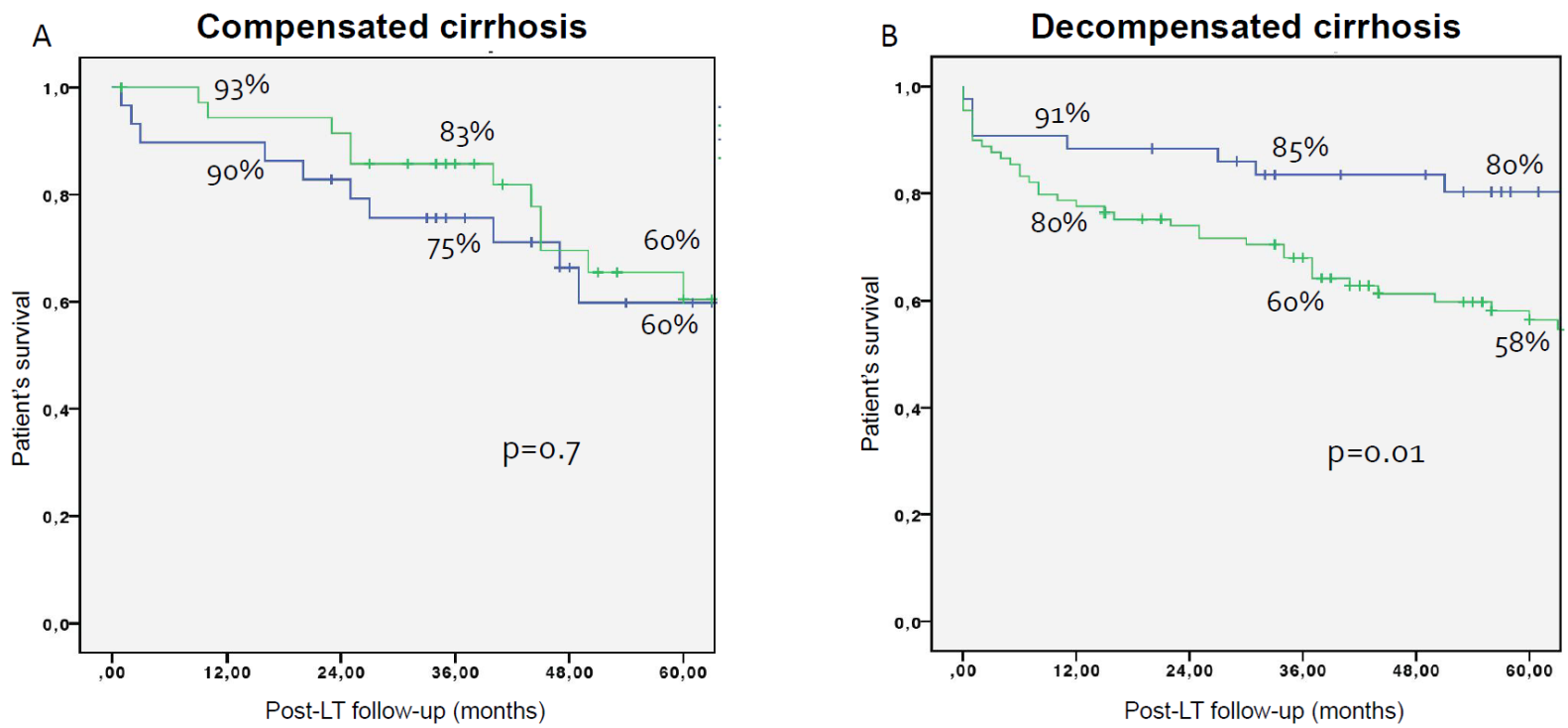

Figure 3. Post-transplant survival in patients with (green line) vs. without (blue line) sarcopenia according to severity of cirrhosis. In compensated patients (Child A), sarcopenia was not associated with reduced survival after transplantation (A); on the other hand, in decompensated patients (Child B/C), sarcopenia was associated with a significantly reduced short- and medium-term survival (B) 

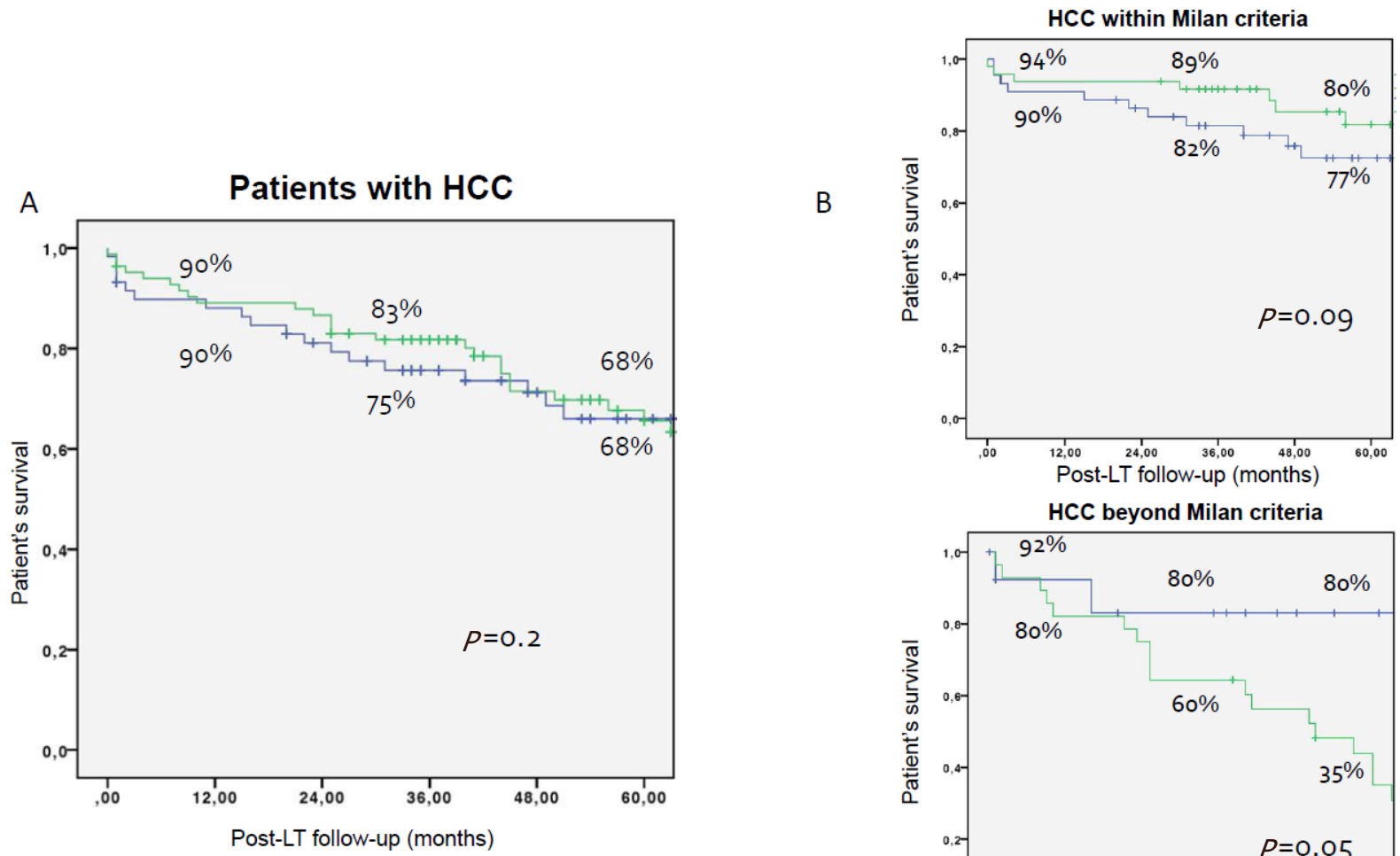

HCC beyond Milan criteria

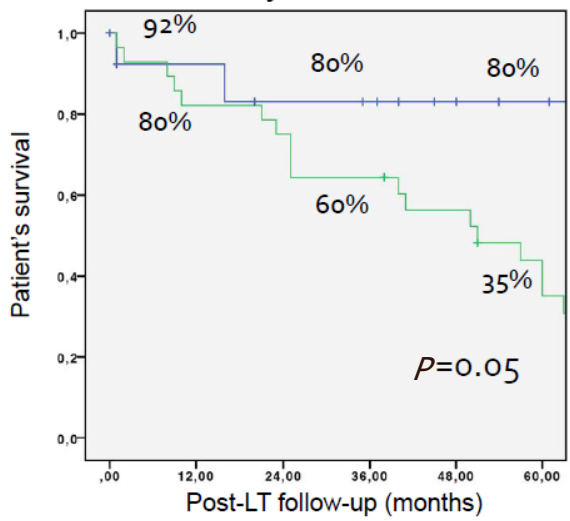

Figure 4. Post-transplant survival in the subgroup of patients with hepatocellular carcinoma (HCC). Overall survival was comparable in patients with (green line) vs. without (blue line) sarcopenia (A); when the analysis was adjusted according to Milan Criteria, sarcopenia was associated with reduced survival in patients with HCC beyond Milan criteria but not in those with HCC within Milan criteria (B)

The analysis was then adjusted per the timing of CT scan prior to LT; and patients with CT scan within 3 months prior to LT $(n=125)$ and those with CT scan between 3 months and 6 months prior to LT ( $n=$ 72) were analysed separately. Interestingly, we found that sarcopenia in was associated with lower survival in decompensated but not in compensated patients in both groups; however in patients with CT scan between 3 months and 6 months, the difference was not as significant $(P=0.05)$ as in those with CT within 3 months prior to LT $(P<0.001)$.

In the subgroup of patients with HCC $(n=142)$, no difference in survival was found between patients with $(n=83)$ and without $(n=59)$ sarcopenia. When the analysis was adjusted for tumour status at the time of transplantation, sarcopenia was associated with a trend towards reduced survival in patients with HCC beyond Milan criteria (28 patients with sarcopenia vs. 14 patients without sarcopenia) but not in patients with HCC within Milan criteria (48 patients with sarcopenia vs. 44 patients without sarcopenia) in whom survival was comparable between the two subgroups [Figure 4]. In patients with HCC beyond Milan criteria, rate of HCC recurrence was slightly higher in patients with HCC beyond Milan criteria with sarcopenia compared to patients without sarcopenia (33\%vs. $22 \%$, respectively); however, the difference was not statistically significant $(P=0.4)$.

Table 2 shows secondary outcomes in patients with $v s$. without sarcopenia. Length of hospitalization, rates of PNF, acute and chronic cellular rejection, biliary complications, and de novo malignancy were not different between patients with and without sarcopenia [Table 2]. On the other hand, bacterial infections were more frequent in patients with compared to those without sarcopenia ( $50 \% v s .35 \% ; P=0.02)$ [Table 2]. 
Table 2. Secondary outcomes in patients with vs. without sarcopenia

\begin{tabular}{|c|c|c|c|}
\hline & $\begin{array}{l}\text { Patients with sarcopenia } \\
\quad(n=122)\end{array}$ & $\begin{array}{l}\text { Patients without sarcopenia } \\
\qquad(n=75)\end{array}$ & $\boldsymbol{P}$ values \\
\hline \multicolumn{4}{|l|}{ Hospitalization (days) } \\
\hline ICU & $6(6)$ & $6.2(6.1)$ & 0.5 \\
\hline Total & $23(19)$ & $24(16)$ & 0.7 \\
\hline $\mathrm{PNF}, n(\%)$ & $4(3 \%)$ & $4(5 \%)$ & 0.4 \\
\hline Bacterial infections, $n(\%)$ & $63(50 \%)$ & $26(35 \%)$ & 0.02 \\
\hline Fungal infections, $n(\%)$ & $13(10 \%)$ & $5(7 \%)$ & 0.3 \\
\hline Viral infections, $n(\%)$ & $11(9 \%)$ & $4(5 \%)$ & 0.3 \\
\hline Biliary stenosis, $n$ (\%) & $23(21 \%)$ & $22(30 \%)$ & 0.1 \\
\hline Acute rejection, $n$ (\%) & $15(12 \%)$ & $8(10 \%)$ & 0.7 \\
\hline Chronic rejection, $n(\%)$ & $1(1.4 \%)$ & $1(2.1 \%)$ & 0.2 \\
\hline De novo malignancy, $n$ (\%) & $4(5.7 \%)$ & $3(6.5 \%)$ & 0.3 \\
\hline
\end{tabular}

Continuous variable expressed as mean (SD), categorical data expressed as frequency and percentage. ICU: intensive care units; PNF: primary non-function

\section{DISCUSSION}

Sarcopenia is a common finding in patients with cirrhosis awaiting liver transplantation ${ }^{[13-18,20,27]}$; however, its impact on post-transplant outcomes remains unclear ${ }^{[19]}$.

Our study shows, in a large retrospective cohort of patients with cirrhosis who underwent liver transplantation, that in the general cohort, sarcopenia is not associated with reduced post-transplant survival. On the other hand, when the analysis was adjusted for severity of cirrhosis (i.e., compensated and decompensated patients analysed separately), we found that patients with decompensated cirrhosis with sarcopenia had a significantly lower survival than controls with decompensated cirrhosis without sarcopenia. By contrast, this effect was not observed in patients with compensated cirrhosis, in whom survival rates were similar between sarcopenic and non-sarcopenic patients. It may be that in sarcopenic patients who undergo liver transplant with compensated liver disease, the negative effect of sarcopenia is relatively less important compared to other factors such as recurrence of primary liver disease. An alternative explanation could be that in compensated patients after LT, there is a more rapid or more significant improvement in muscle mass after LT compared with those who undergo transplantation with decompensated cirrhosis, which prevents the negative sequalae associated with sarcopenia. Further studies that look at the changes of muscle mass after transplantation in compensated $v s$. decompensated patients at the time of transplantation are required to test this hypothesis.

These findings suggest that the impact of sarcopenia on post-transplant survival may vary significantly according to the severity of liver dysfunction at time of transplantation and that proactive treatment of pretransplant sarcopenia should be especially considered in decompensated candidates in whom improvement of muscle mass could potentially translate into improvement in post-transplant survival.

Some studies have previously assessed the effect of pre-transplant sarcopenia on the risk of complications and survival after transplantation, and have reported conflicting results with sarcopenia being associated with increased risk of death in some studies but not in others ${ }^{[14,16-18,27,28]}$. In fact, in a very recent metaanalysis, van Vugt et al. ${ }^{[12]}$ suggested that the current evidence is not robust enough to support the association between sarcopenia and increased risk of death after transplantation. While awaiting large prospective studies to evaluate the impact of pre-transplant sarcopenia on post-transplant mortality, our findings suggest that the severity of liver dysfunction at the time of transplantation is a key factor in this analysis and it should be taken into consideration when assessing the clinical impact of sarcopenia in this patient population. 
There is a lack of agreement on how to assess skeletal muscle abnormalities in patients with cirrhosis ${ }^{[2]}$. Per our protocol, sarcopenia was defined by direct quantification of skeletal muscle mass at cross-sectional imaging (CT scan), which is currently considered the gold standard in patients with cirrhosis due to its objective and reproducible measurements. In addition, among the different CT scores that can be used to assess sarcopenia, we specifically choose L3 SMI because of its good correlation with whole body skeletal muscle mass ${ }^{[3,30]}$.

In addition, we used sex-specific cut-offs previously proposed for the diagnosis of sarcopenia in patients with cirrhosis awaiting $\mathrm{LT}^{[3]}$, and we included only patients with a radiological assessment within the 6 months prior to transplantation. Because the muscle mass in patients with cirrhosis may significantly change over a 6 months period, we have assessed the impact of sarcopenia in decompensated patients with CT scan within 3 months prior to LT and in patients with CT scan between 3 months and 6 months prior to LT separately. Interestingly, we found that sarcopenia was associated with lower survival in decompensated but not in compensated patients in both groups; however, the difference in patients with CT scan between 3 months and 6 months was not as evident as in those with CT scan within 3 months, which would suggest that to evaluate the impact of sarcopenia on post-transplant outcomes the evaluation of muscle mass should be performed as close as possible to transplantation.

In line with previous data ${ }^{[3]}$, we confirm the high prevalence of sarcopenia in patients with cirrhosis awaiting LT as well as the positive correlation between sarcopenia and increasing severity of liver dysfunction (Child C > Child A), particularly in males ${ }^{[6]}$. Interestingly enough, however, approximately $50 \%$ of patients with Child A included in our study were sarcopenic, which indicates that sarcopenia should be actively screened in all patients with cirrhosis evaluated for transplantation, independent of liver dysfunction severity. On the same note, we found no association between prevalence of sarcopenia and patient sex (60\% in male and 50\% in female patients), aetiology of liver disease (i.e., patients with HCV, alcoholic, and metabolic-related liver disease), or presence of HCC. This further suggests that the assessment of sarcopenia should be performed in any patient evaluated for LT, independent of sex, aetiology, and indication for transplant ${ }^{[31-33]}$; however this would need confirmation due to the relatively small sample size in our study.

Since the majority of patients included in our analysis had HCC, we also sought to determine the role of sarcopenia in this patient population. In our center, evaluation of transplantability and wait-list priority in candidates with HCC is not based on morphological criteria, such as Milan criteria, but on other factors that would reflect tumour behaviour and aggressiveness, including response to downstaging/ bridging treatments and characteristics and timing of HCC recurrence after treatment ${ }^{[34]}$. Thus, we were able to include patients transplanted with HCC beyond MC and to evaluate in these patients the impact of sarcopenia.

We found a weak association between tumour size, as defined by Milan criteria, and prevalence of sarcopenia (being higher in patients with HCC beyond $v s$. within Milan criteria).

On the same note, we noticed that sarcopenia was associated with worse post-LT survival only in patients who underwent transplantation with HCC beyond Milan criteria. It has been suggested that sarcopenia and alterations of body composition may be associated with increased risks of HCC recurrence and death after transplantation ${ }^{[28,35,36]}$. Our data would further suggest that the impact of sarcopenia in this patient population may vary according to the tumour size, and that patients with more advanced HCC are probably the most at risk.

Among secondary outcomes, we found that sarcopenia was associated with a higher rate of early bacterial infection after transplantation, with as many as $50 \%$ of sarcopenic recipients having at least one infection 
in the early post-transplantation period. In agreement with previous finding $\mathrm{s}^{[19]}$, these findings suggest that patients with sarcopenia are particularly vulnerable to bacterial infections ${ }^{[19,37,38]}$ and that specific antibiotic prophylaxis may be considered in these patients during the early period post-transplantation.

Our study has some limitations. Firstly, due to the retrospective design, only association and not causation could be determined. Furthermore, some important variables such as donor and graft characteristics, surgery-related factors, causes of death, and specific data on food intake or nutritional intervention before or after LT were lacking. Thus, our findings require validation by large prospective cohorts. Secondly, the most common indication for LT in our cohort was HCV-related cirrhosis and most patients were transplanted before the introduction of direct acting antivirals ${ }^{[39]}$. Now that the widespread adoption of interferon-free antiviral treatments has significantly changed the composition of the wait-list as well as post-transplant outcomes in HCV recipients ${ }^{[40-42]}$, new studies looking at the effect of sarcopenia are needed.

In conclusion, we show that sarcopenia is a common finding in patients with cirrhosis awaiting transplantation, independent of sex, indication for LT, aetiology, and severity of underlying liver disease. In those who undergo transplantation with decompensated cirrhosis, sarcopenia is associated with a significantly reduced post-transplant survival.

The assessment of sarcopenia in liver transplant candidates as well as its proactive treatment may improve the recipient's outcome and should be considered in all patients with cirrhosis awaiting transplantation, particularly those who are decompensated.

\section{DECLARATIONS}

\section{Authors' contributions}

Research design, performance of the research, and writing of the manuscript: D’Arcangelo F, Zanetto A Research design and collection of the data: Aliberti C, Shalaby S, Pellone M, Sciarrone SS, Becchetti C, Ferrarese A, Gambato M, Russo FP, Germani G, Senzolo M, Vitale A, Cillo U

Research design, critical revision and final approval of the manuscript: Burra $\mathrm{P}$

\section{Availability of data and materials}

Not applicable.

\section{Financial support and sponsorship}

None.

\section{Conflicts of interest}

All authors declared that there are no conflicts of interest.

\section{Ethical approval and consent to participate}

This was a single-center, retrospective, case-control study approved by the Padua University Hospital Ethical Committee (\#AOP/0564). The study was conducted in compliance with the Declaration of Helsinki and a waiver for informed consent was obtained (retrospective chart review).

\section{Consent for publication}

Not applicable.

\section{Copyright}

(c) The Author(s) 2021. 


\section{REFERENCES}

1. Cruz-Jentoft AJ, Bahat G, Bauer J, et al; Writing Group for the European Working Group on Sarcopenia in Older People 2 (EWGSOP2), and the Extended Group for EWGSOP2. Sarcopenia: revised European consensus on definition and diagnosis. Age Ageing 2019;48:16-31.

2. Bhanji RA, Narayanan P, Moynagh MR, et al. Differing impact of sarcopenia and frailty in nonalcoholic steatohepatitis and alcoholic liver disease. Liver Transpl 2019;25:14-24.

3. Carey EJ, Lai JC, Wang CW, et al; Fitness, Life Enhancement, and Exercise in Liver Transplantation Consortium. A multicenter study to define sarcopenia in patients with end-stage liver disease. Liver Transpl 2017;23:625-33.

4. Lai JC, Dodge JL, Sen S, Covinsky K, Feng S. Functional decline in patients with cirrhosis awaiting liver transplantation: results from the functional assessment in liver transplantation (FrAILT) study. Hepatology 2016;63:574-80.

5. Stirnimann G, Ebadi M, Tandon P, Montano-Loza AJ. Should sarcopenia increase priority for transplant or is it a contraindication? Curr Gastroenterol Rep 2018;20:50.

6. Tandon P, Ney M, Irwin I, et al. Severe muscle depletion in patients on the liver transplant wait list: its prevalence and independent prognostic value. Liver Transpl 2012;18:1209-16.

7. van Vugt JLA, Alferink LJM, Buettner S, et al. A model including sarcopenia surpasses the MELD score in predicting waiting list mortality in cirrhotic liver transplant candidates: a competing risk analysis in a national cohort. $J$ Hepatol 2018;68:707-14.

8. van Vugt JLA, Buettner S, Alferink LJM, et al. Low skeletal muscle mass is associated with increased hospital costs in patients with cirrhosis listed for liver transplantation-a retrospective study. Transpl Int 2018;31:165-74.

9. Yadav A, Chang YH, Carpenter S, et al. Relationship between sarcopenia, six-minute walk distance and health-related quality of life in liver transplant candidates. Clin Transplant 2015;29:134-41.

10. Ebadi M, Montano-Loza AJ. Clinical relevance of skeletal muscle abnormalities in patients with cirrhosis. Dig Liver Dis 2019;51:1493-9.

11. Pinto Dos Santos D, Kloeckner R, Koch S, et al. Sarcopenia as prognostic factor for survival after orthotopic liver transplantation. Eur $J$ Gastroenterol Hepatol 2020;32:626-34.

12. van Vugt JL, Levolger S, de Bruin RW, van Rosmalen J, Metselaar HJ, IJzermans JN. Systematic review and meta-analysis of the impact of computed tomography-assessed skeletal muscle mass on outcome in patients awaiting or undergoing liver transplantation. $A m J$ Transplant 2016;16:2277-92.

13. Aby ES, Lee E, Saggi SS, et al. Pretransplant sarcopenia in patients with NASH cirrhosis does not impact rehospitalization or mortality. $J$ Clin Gastroenterol 2019;53:680-5.

14. DiMartini A, Cruz RJ Jr, Dew MA, et al. Muscle mass predicts outcomes following liver transplantation. Liver Transpl 2013;19:1172-80.

15. Engelmann C, Schob S, Nonnenmacher I, et al. Loss of paraspinal muscle mass is a gender-specific consequence of cirrhosis that predicts complications and death. Aliment Pharmacol Ther 2018;48:1271-81.

16. Englesbe MJ, Patel SP, He K, et al. Sarcopenia and mortality after liver transplantation. J Am Coll Surg 2010;211:271-8.

17. Golse N, Bucur PO, Ciacio O, et al. A new definition of sarcopenia in patients with cirrhosis undergoing liver transplantation. Liver Transpl 2017;23:143-54.

18. Montano-Loza AJ, Meza-Junco J, Baracos VE, et al. Severe muscle depletion predicts postoperative length of stay but is not associated with survival after liver transplantation. Liver Transpl 2014;20:640-8.

19. Ooi PH, Hager A, Mazurak VC, et al. Sarcopenia in chronic liver disease: impact on outcomes. Liver Transpl 2019;25:1422-38.

20. Underwood PW, Cron DC, Terjimanian MN, Wang SC, Englesbe MJ, Waits SA. Sarcopenia and failure to rescue following liver transplantation. Clin Transplant 2015;29:1076-80.

21. Burra P, Belli LS, Ginanni Corradini S, et al. Common issues in the management of patients in the waiting list and after liver transplantation. Dig Liver Dis 2017;49:241-53.

22. Burra P, Giannini EG, Caraceni P, et al. Specific issues concerning the management of patients on the waiting list and after liver transplantation. Liver Int 2018;38:1338-62.

23. Garcia-Tsao G, Abraldes JG, Berzigotti A, Bosch J. Portal hypertensive bleeding in cirrhosis: Risk stratification, diagnosis, and management: 2016 practice guidance by the American Association for the study of liver diseases. Hepatology 2017;65:310-35.

24. Zanetto A, Garcia-Tsao G. Management of acute variceal hemorrhage. F1000Res 2019;8:966.

25. European Association for the Study of the Liver. EASL Clinical Practice Guidelines: Management of hepatocellular carcinoma. J Hepatol 2018;69:182-236.

26. Mazzaferro V, Regalia E, Doci R, et al. Liver transplantation for the treatment of small hepatocellular carcinomas in patients with cirrhosis. N Engl J Med 1996;334:693-9.

27. Kalafateli M, Mantzoukis K, Choi Yau Y, et al. Malnutrition and sarcopenia predict post-liver transplantation outcomes independently of the model for end-stage liver disease score. J Cachexia Sarcopenia Muscle 2017;8:113-21.

28. Valero V 3rd, Amini N, Spolverato G, et al. Sarcopenia adversely impacts postoperative complications following resection or transplantation in patients with primary liver tumors. J Gastrointest Surg 2015;19:272-81.

29. Becchetti C, Bosch J. Muscle abnormalities in cirrhosis: calling for more strength in evaluation and prevention. Dig Liver Dis 2019;51:1500-1.

30. Ebadi M, Wang CW, Lai JC, et al; From the fitness, life enhancement, and exercise in liver transplantation (FLEXIT) Consortium. Poor performance of psoas muscle index for identification of patients with higher waitlist mortality risk in cirrhosis. $J$ Cachexia Sarcopenia Muscle 2018;9:1053-62.

31. European Association for the Study of the Liver. EASL Clinical Practice Guidelines: liver transplantation. J Hepatol 2016;64:433-85.

32. European Association for the Study of the Liver. EASL Clinical Practice Guidelines for the management of patients with decompensated 
cirrhosis. J Hepatol2018;69:406-60.

33. European Association for the Study of the Liver. EASL Clinical Practice Guidelines on nutrition in chronic liver disease. $J$ Hepatol 2019;70:172-93.

34. Cillo U, Burra P, Mazzaferro V, et al. A multistep, consensus-based approach to organ allocation in liver transplantation: toward a "blended principle model". Am J Transplant 2015;15:2552-61.

35. Chang KV, Chen JD, Wu WT, Huang KC, Hsu CT, Han DS. Association between loss of skeletal muscle mass and mortality and tumor recurrence in hepatocellular carcinoma: a systematic review and meta-analysis. Liver Cancer 2018;7:90-103.

36. Grąt K, Pacho R, Grąt M, Krawczyk M, Zieniewicz K, Rowiński O. Impact of body composition on the risk of hepatocellular carcinoma recurrence after liver transplantation. J Clin Med 2019;8:1672.

37. Kim G, Kang SH, Kim MY, Baik SK. Prognostic value of sarcopenia in patients with liver cirrhosis: a systematic review and metaanalysis. PLoS One 2017;12:e0186990.

38. Lucidi C, Lattanzi B, Di Gregorio V, et al. A low muscle mass increases mortality in compensated cirrhotic patients with sepsis. Liver Int 2018;38:851-7.

39. Toniutto P, Zanetto A, Ferrarese A, Burra P. Current challenges and future directions for liver transplantation. Liver Int 2017;37:317-27.

40. Ferrarese A, Germani G, Gambato M, et al. Hepatitis C virus related cirrhosis decreased as indication to liver transplantation since the introduction of direct-acting antivirals: a single-center study. World J Gastroenterol 2018;24:4403-11.

41. Ferrarese A, Zanetto A, Gambato M, et al. Liver transplantation for viral hepatitis in 2015. World J Gastroenterol 2016;22:1570-81.

42. Russo FP, Ferrarese A, Zanetto A. Recent advances in understanding and managing liver transplantation. F1000Res 2016;5:2895. 